to the 'inappropriate GP referral' we so often complain about.

My trainer was an older GP, who had worked in the practice for many years. His knowledge of, and relationships with, many families under his care was impressive and I realised how often we miss out on tapping in to such a resource by poor liaison with the family doctor.

The post was not always enjoyable; I found the endless 'coughs and colds' monotonous, the winter 'flu epidemic of 1990 (which increased home visits threefold) stressful, and was irritated by spending seemingly endless hours on the telephone trying to arrange an acute medical or surgical admission. At the end of my time, I finally decided on psychiatry as the career I would definitely pursue. However, overall I gained so much and hope there will continue to be similar opportunities avallable for other interested psychiatric trainees in the future.

H.D. Foster, South Western Hospital, Pulross Road, London SW9

\section{GPs' attitudes towards sectorisation}

Sir: I was disappointed to read Dr Chaudhary's account of the unsuccessful attempts of his community care NHS trust to sectorise their service (Psychiatric Bulletin, May 1994, 18, 308).

We are all becoming aware of the importance of involving GPs in decisions about the way the services are delivered and the need for increased communication. While in the past there has been an acknowledgement of this issue, it has assumed greater importance with the changes in funding arrangements. In my own experience the attitude of GPs towards proposals for sectorisation is often negative, but in practice their concerns tend not to be borne out. For the GP there is the loss of the ability to make referrals not only to different consultants but also to choose different mental health professionals. This can lead to unnecessary duplication while the sectorised team forms a filter mechanism for allocation to the most appropriate discipline. Given the up to 20-fold variation in referral levels between GPs and the varying ability and interest in psychiatry, the failure to sectorise tends to compound the existing inefficiencies in the way our services are provided. The task seems to be to convince GPs that the potential disadvantages from their perspective are far outweighed by the gains to the overall service provided for their patients.

HARRY DOYLE, Northwick Park Hospital, Harrow HA1 3UJ

\section{Surviving as an overseas doctor}

Sir: I was interested to read Swaran Singh's advice for overseas doctors (Psychiatric Bulletin,
May 1994, 18, 302-303), in which he sets out what to do and not to do when working in a strange and new culture. In a second paper (Singh, 1994) he describes in more harrowing terms some of the difficulties he has faced as a foreign doctor in England. For the past 18 months I have been working in Hong Kong, and as a foreign, overseas doctor in this very different setting, I can identify with many of his problems, from getting used to the food, to adjusting to the way different professionals work together.

The most obvious difficulty faced by a foreign doctor is that of language. In my case, I am still not able to speak the same language as most of my patients and rely on medical students, junior doctors or nurses for translation. I have found it surprisingly easy to use untrained interpreters in assessing a patient's mental state, but there are occasions in which my inability to speak the same language as a patient has been frustrating.

The authority of doctors was also something that was new to me. As a white doctor, a 'foreign devil', I am regarded by some patients as even more of an authority figure than a Chinese doctor. This hierarchy extends to team members, thus interactions between disciplines are more formal than in England and decisions are almost always made by the medical staff.

Overseas doctors, wherever they go or wherever they are from, can expect to have to spend several months adjusting. There are language problems, cultural problems and a new way of working to learn. The patients themselves are remarkably similar, given the completely different backgrounds, culture, and language that they have. I have found this experience rewarding and feel that it has broadened my outlook both within psychiatry and without.

SingH. S.P. (1994) Cultural adjustment and the overseas trainee. British Medical Journal. 308, 1169.

HELEN BAXTER, Department of Psychiatry, Chinese Untversity of Hong Kong. Prince of Wales Hospital, Shatin. Hong Kong

\section{Fragmentation of community services}

Sir: Drs Fear and Cattell (Psychiatric Bulletin, May 1994, 18, 263-265) comment on the fragmentation of essential community services with the advent of multiple purchasers who can dictate where their patients receive care.

I would urge the NHS to look carefully at these developments, as not only will you see fragmentation, but ultimately fewer services may be available. In many parts of Canada there are no defined catchment areas for general hospitals and no co-ordination of services. Many towns have at least two hospitals (originally Protestant and Catholic). Our own city has three general 